\title{
A SECTORED RECEIVER FOR INFRARED WIRELESS NETWORKS
}

\author{
Luís Nero Alves ${ }^{\Sigma \Lambda}$, Rui L. Aguiar ${ }^{\Sigma}$, Eduardo de Vasconcelos $^{\Sigma}$, José Luís Cura ${ }^{\Sigma}$ \\ ${ }^{\Sigma}$ Dpt. Electrónica e Telecomunicações, Universidade de Aveiro / Instituto de Telecomunicações \\ Campus de Santiago, 3810 Aveiro, Portugal, Tel: +351.34.370200, Fax: +351.34.381128. \\ ${ }^{\Lambda}$ Escola Superior de Tecnologia e Gestão, Instituto Politécnico de Leiria, Morro do Lena - Alto Vieiro, \\ 2400 Leiria, Portugal, Fax: +351.44.820310. \\ Email:nero@av.it.pt ruilaa@det.ua.pt jic@det.ua.pt edvasc@av.it.pt
}

\begin{abstract}
This paper presents an experimental sectored receiver for infrared wireless networks. The receiver comprises two sectors, each with a switched gain front-end and a signal-to-noise ratio estimator. These are then interconnected with a best-sector selector unit, able to compensate the gain switching characteristics of the front-ends. The circuit has been designed in a $0.8 \mu \mathrm{m}$ CMOS technology.
\end{abstract}

\section{INTRODUCTION}

Optical wireless communications have gone through large developments in past years, as optical transducer technology (mainly infrared, in this case) has decreased its price. Due to this emergent wireless LAN technology, and to the promising infrared medium, several efforts have been made in the design of optical receivers [1-2]. The key aspect of these optical receivers is their "almost" diffuse nature of reception. This requires circuits with very flexible characteristics of gain (large sensitivity) and large

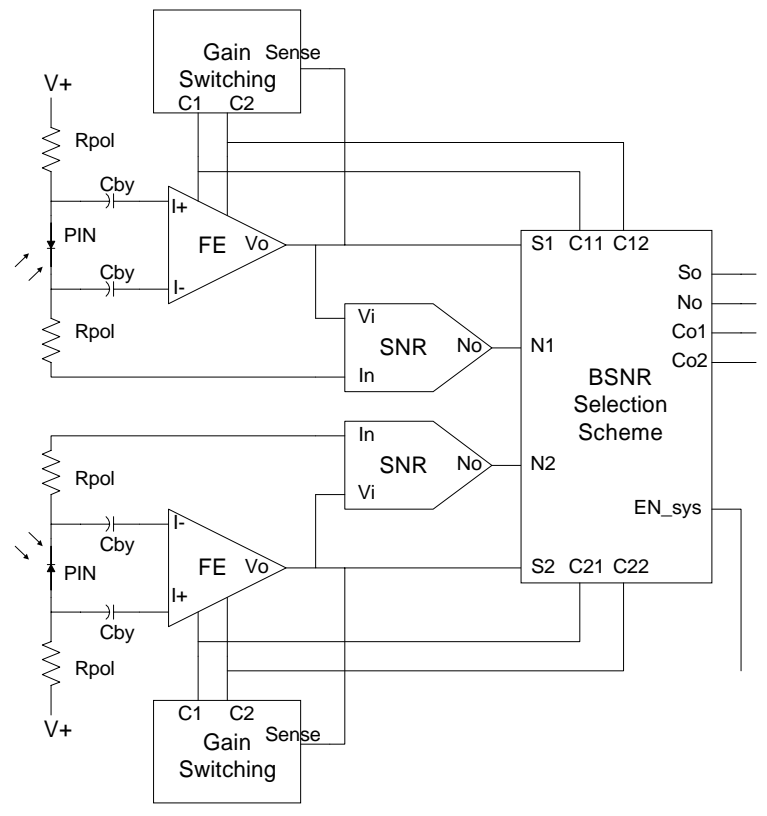

Fig. 1 - Overall circuit input dynamic range. Furthermore, as dominant noise sources are usually fixed (such as illumination lamps or windows), the optical sensor is preferably composed of several photo-detectors with a small FOV, oriented in different directions [3]. Thus most of the surrounding environment area will be covered but some of the principal noise sources will still be avoided. The receiver is then responsible for processing the information from all photo-detectors in order to maximise signal while minimising noise. With Best Sector receivers only the sector with the best signal-to-noise ratio (SNR) is selected. This contrasts with the case of a Maximal-Ratio receiver, where the output signals of all sectors are combined through an adder circuit, and each output signal is proportional to its respective SNR.

This paper describes a $0.8 \mu \mathrm{m}$ CMOS circuit comprising a twosector receiver, each with a large gain-bandwidth product (larger than $20 \mathrm{THz} \Omega$, for a $10 \mathrm{pF}$ input photodiode) and a signal-to-noise estimator targeted for the expected input dynamic ranges. This information is fed on a processing unit, able to compensate SNR evaluation distortions introduced by gain switching, and to select the sector with the best SNR.

\section{SYSTEM OVERVIEW}

The target system (Fig. 1) comprises two equal paths beginning in two different photo-detectors, two front-end transimpedance amplifiers, and two circuits for SNR measurement. The circuit responsible for the implementation of the adequate sectorization strategy is also shown. In our case we have chosen to use a Best Sector receiver, due to the complexity of implementing maximalratio techniques with switched gain transimpedance amplifiers. In Fig. 1 the photodiode polarization and bypass components are also shown.

\subsection{Optical Front-end}

The implemented Front-end consists of three stages: a differential transimpedance amplifier with a switched feedback network, a differential to single-ended voltage amplifier and an output buffer, as shown in Fig. 2. The three stages are DC coupled to avoid baseline shift with signal input. Additionally a control block is added for gain switching purposes, with small variations of the circuit described in [4]. 


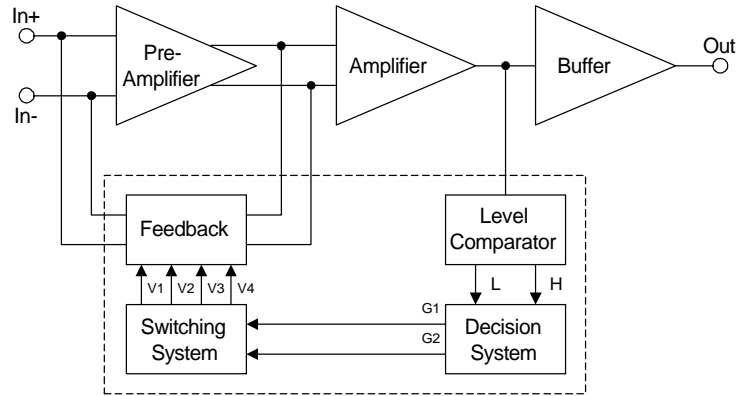

Fig. 2 - Block diagram of the front-end.

Electromagnetic interference is a major system issue for the desired gain values. For its minimization, the circuit has a differential input, to which low cost photodiodes $(10 \mathrm{pF}$ to $50 \mathrm{pF}$ junction capacitance) will be connected in the targeted wireless system. Thus, for noise minimization considerations the transimpedance amplifier also presents an input stage with an equivalent $10 \mathrm{pF}$ capacitance [4]. Input referred current noise was under $8.2 \mathrm{pA} / \sqrt{\mathrm{Hz}}$.

The input transimpedance amplifier was implemented with a step gain switched feedback network, achieving a $60 \mathrm{~dB}$ dynamic range. This approach can be safely used, as maximum optical signal fading (under $15 \mathrm{~dB}$ ) is not sufficient to produce gain switching oscillations.

\subsection{SNR measurement circuit}

The SNR measurement circuit is responsible for the dynamic calculation of the signal to noise measurement at the front-end's input. This signal to noise ratio is proportional to the ratio of the average power of the input signal $\left(I_{i}\right)$ and average (optical) noise power presented in the photo-detector, $\left\langle I_{i}\right\rangle / \sigma$. This noise current is roughly proportional to the photodiode current. In our circuit we implemented the ratio of the front-end's squared amplitude to the squared noise, $\left\langle\mathrm{V}_{o}\right\rangle^{2} / \sigma^{2}$, where $V_{o}$ is proportional to $I_{i}$ (at the

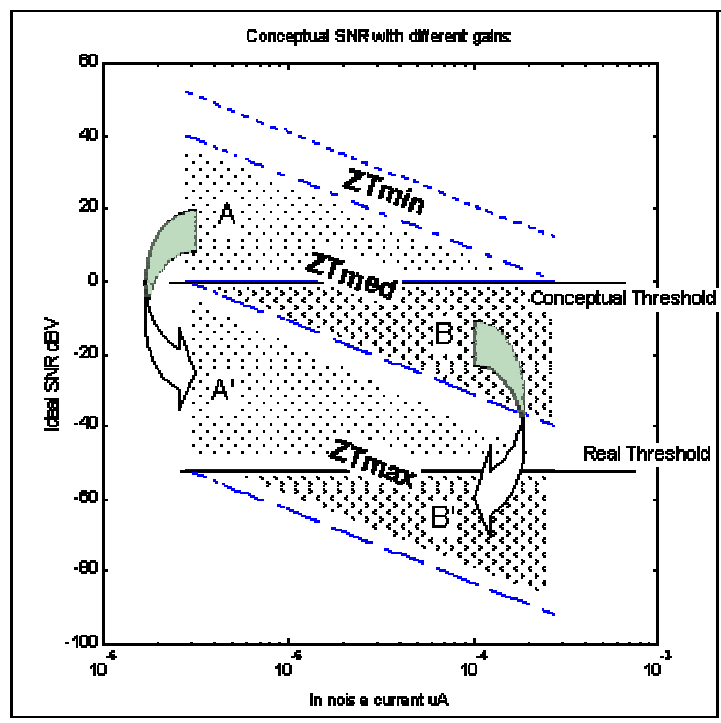

Fig. 4 - Conceptual SNR correction scheme. ZTi are the switched transimpedance gains of the front-ends

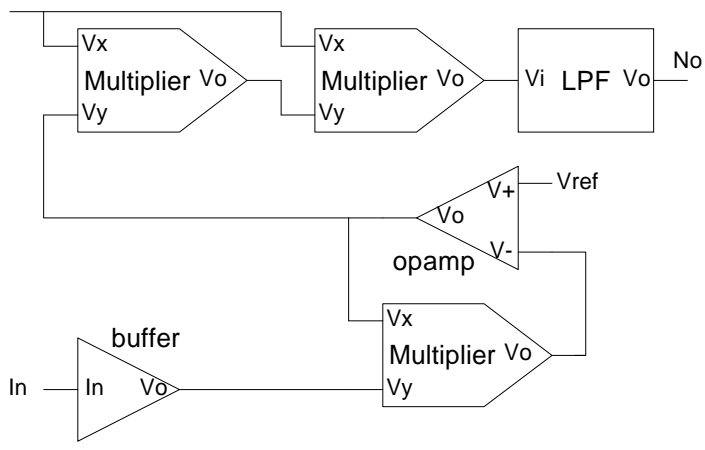

Fig. 3 - SNR measurement Circuit

output of the front-end) and $\sigma^{2}=2 q I_{n} B$ ( $q$ is the charge of the electron, $I_{n}$ is the noise current in the photo-detector and $B$ the bandwidth).

The circuit for SNR measurement is presented in fig. 3, with two multipliers and one divider (implemented with a multiplier in a feedback loop). The divider is used to execute the necessary division $\left(1 / I_{n}\right)$ and the two multipliers are used to achieve the squared SNR result. The last stage in Fig. 3 is a low pass filter necessary to implement the desired "average" measure of the SNR. Notice also that the buffer at the $I_{n}$ input is a transimpedance amplifier used to sense the photo-detector noise current.

All the necessary local voltage references are generated inside the chip. This point is of particular importance for the multipliers, which need a dynamic polarization scheme, for improved resilience to signal level variations [5].

\subsection{Sectorization and SNR correction}

The sectorization scheme used in this circuit was a Best Sector approach. The selected sector is the one that exhibits the best possible SNR at a given point in time. The major problem in the design of the best sector selection circuit was the fact that the transimpedance amplifier can exhibit three different gains according to its gain switch capability. So for our concern, we would have three different bands of possible measured SNR's. Fig. 4 represents these three bands and what real happens when the circuit works. These three bands (due to the three transimpedance gains of the front-ends, ZTmin, ZTmed and ZTmax) became a single output band and the only way to recognize them is through the gain switching signals generated in the front-end $(\mathrm{C} 1, \mathrm{C} 2$, in Fig. 1). Fig. 5 represents the implemented circuit. Six different situations are relevant for signal selection, and are mapped in two distinct areas (in Fig. 4, only four of these areas are highlighted, as well as its mapping into working areas A' and B'). The correct choice of the best sector is accomplished in the following way: i) if the two sectors have the same gain, the choice falls directly on the best SNR of the two sectors. ii) If the two sectors have different gains, the choice falls on the sector with minimum gain if its SNR is superior to a given threshold, dependent on the front-end characteristics (SNRT), otherwise is the best of the SNR's. The key aspects of this circuit (Fig. 5) are the two comparators needed: the first compares the two SNR's (N1 and N2) of each sector and chooses the best (BSNR signal); the second compares the SNR of the sector with minimum gain with the threshold SNRT and produces the EN_BSNR signal. This signal acts as an enable to the 


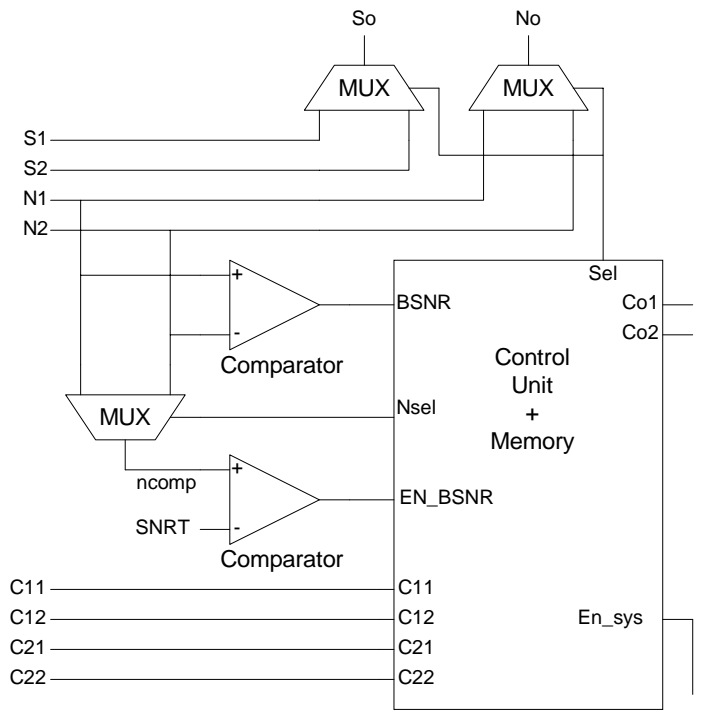

Fig. 5 - Best Sector integration

BSNR one, defining the comparison situation the system is in. The external signal En_sys allows some control over the selection scheme, as it may be desirable to establish one sector independent of the measured SNR for test purposes. The outputs of the selector are: the best signal (So), the SNR (No) and the gain switch signals (Co1, Co2) of the chosen sector.

\section{RESULTS}

This circuit was implemented with a standard double metal, double poly, $0.8 \mu \mathrm{m}$ CMOS. The next three sub-sections show some of the results achieved, for typical wireless (low cost, $4 \mathrm{Mb} / \mathrm{s}$ ) LAN applications. Power consumption is in the range of $150 \mathrm{~mW}$ and the layout area of $1600 \mu \mathrm{m} \times 2000 \mu \mathrm{m}$.

\subsection{Front-end}

The measured frequency response results shown in Fig. 6 were achieved with a (typical) $50 \mathrm{pF}$ parasitic capacitance photodiode

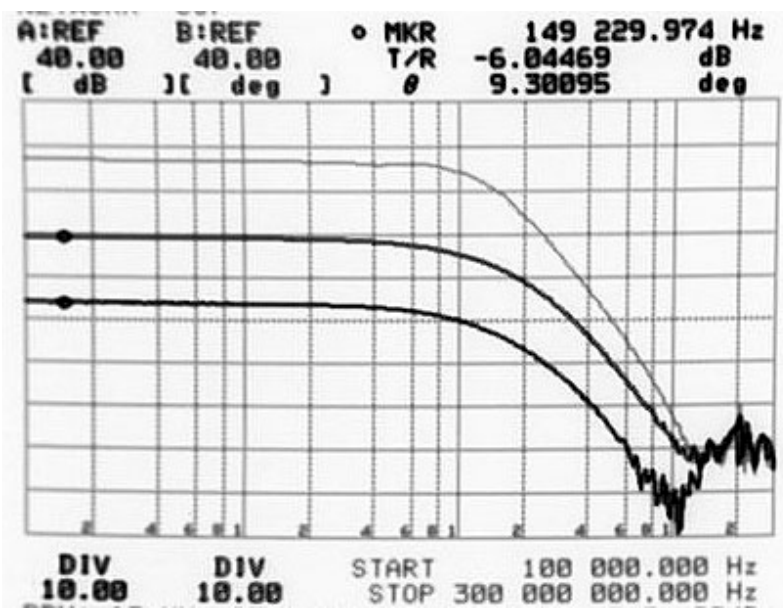

Fig. 6 - Front-end frequency response (three gains) and show a better than $10 \mathrm{MHz}$ bandwidth, and a maximum gain larger than $400 \mathrm{~K} \Omega$ (lower gains of $40 \mathrm{~K} \Omega$ and $8 \mathrm{~K}$ ). These values exceed the requirements for the low-cost wireless LAN we are developing.

\subsection{SNR measurement}

The implemented multiplier used a folded Gilbert strategy with dynamic bias scheme. Simulation results showed a better than $20 \mathrm{MHz}$ bandwidth, a larger than $20 \mathrm{~dB}$ input range, and an output excursion that can span over $50 \mathrm{~dB}$. The divider uses a multiplier in the feedback loop of an operational amplifier, and achieves 50dB of input and output dynamic ranges, with a $2 \mathrm{MHz}$ imposed bandwidth [5]. These results were appropriate for the expected dynamic ranges in wireless optical LANs [6].

Finally, the low pass filter has a cutoff frequency that can be adjusted from the exterior and can span more than one decade of adjustment (from $100 \mathrm{KHz}$ to $1.2 \mathrm{MHz}$ ).

The measured results agree with the simulations. Fig. 7 illustrates the behavior of the SNR measurement circuit, with an input pulsed signal (not showed), and with periodic triangular noise. Fig. 8 shows the SNR measurement output as a function of the input signal and the input noise presented in the photodiode

\subsection{Best Sector integration}

The complete description of the sectorization behavior is lengthy, due to the various units comprising the receiver, and required for the sectorization control unit. Its behavior is exemplified in the simulation presented in figures 9 and 10 , and has been demonstrated experimentally. Fig. 9 shows the input signals applied to the selector circuit: the measured SNR of both sectors $(n 1, n 2)$, the signals from both sectors $(s 1, s 2$; where we choose sector 2 to have a fixed gain and sector 1 to switch its gain according to the signal level), the gain switch control signals (where we can be see the fixed gain of sector $2-c 21$ and $c 12$ - and the gain switching in sector $1-$ signals $c 11$ and $c 12-$ ), and finally the system enable (En_sys) signal, being turned on and off, simulating the reception of consecutive packets. The input signals were chosen in such a way that the best SNR is changing from sector 1 to sector 2. (The black stripes on $s 1$ and $s 2$ are due to visualization resolution, as these are pulsed quasi-periodic signals, much faster than noise variations). Fig. 10 shows the results of the selection circuit. Due to the example we have chosen, our signals will have a fixed level present in the En_BSNR signal (fig. 5). Thus the principal decision signal will be the output of the best SNR

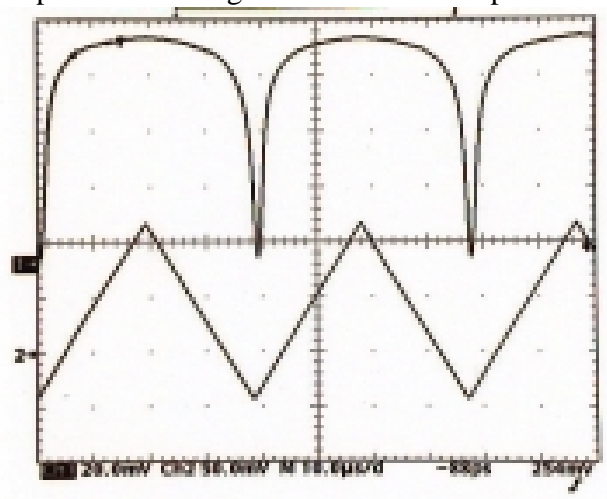

Fig. 7 - SNR measurement transient response 


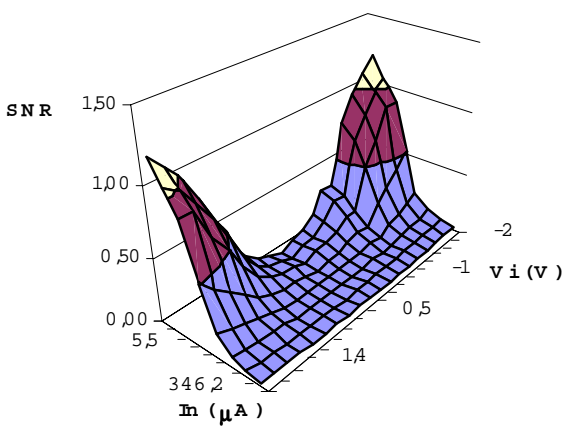

Fig. 8 - SNR transfer function (absolute values)

comparator $(B S N R)$. The ncomp signal represents the SNR of the sector with minimum gain; note that some variations are present due to gain switching in sectorl and the signal $(s l)$ changes imposed to the same sector. The Sel signal is very similar to the $B S N R$ signal but for some of the edges, where the system is inhibited by En_sys. The rest of the signals represent the selected gain switching signals (Col, $\mathrm{Co} 2$ ), the best SNR value (no) and the output signal (so) of the best sector (which varies between sector 1 and sector 2 , as can be easily seen by the waveforms). The output signal shows an almost uniform level, due to the best selection scheme that tends to regulate the signal level in this example: this may not happen with other situations.

\section{CONCLUSIONS}

A two-sector receiver for infrared wireless networks has been described. This receiver is able to implement a Best Sector approach, using switched gain high-performance front-ends, and

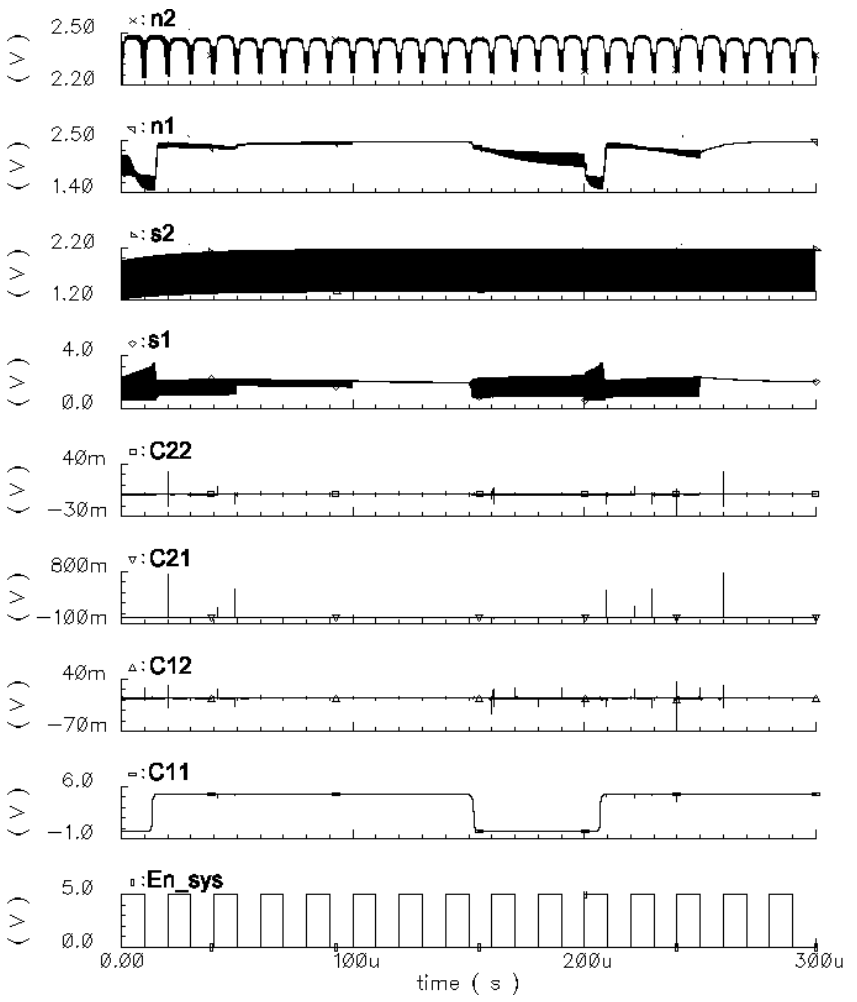

Fig. 9 - Sectorization: inputs compensating for the gain switching. The circuit is expected to be able to work with signal levels as low as $100 \mathrm{nA}$, in optical noise levels orders of magnitude above, and to be able to chose the signal with the best SNR. This circuit can be easily expanded to support a larger number of input sectors.

\section{REFERENCES}

[1] J.M. Khan, J.R. Barry, "Wireless Infrared Communications", Proc. of the IEEE, Vol. 85, N², Feb. 97.

[2] R.L. Aguiar, A.M. Tavares, J.L. Cura, E. de Vasconcelos, L.N. Alves, R.T. Valadas, D.M. Santos, "Considerations on the design of transceivers for wireless optical LANs", IEE Electronics \& Communications, Colloquium on Optical Wireless Communications, London, England, Jun 99.

[3] R.T. Valadas, A.M. Tavares, A. de Oliveira Duarte, "Angle Diversity to Combat Ambient Noise in Indoor Optical Wireless Communication Systems", Int. J. Wireless Information Networks, Vol. 4, No 4, 1997.

[4] E. Vasconcelos, J.L. Cura, R.L. Aguiar, D.M.Santos, "A Novel High-Gain, High Bandwidth CMOS Differential FrontEnd for Wireless Optical Systems", ISCAS'99, Orlando, Florida, May 1999, pp. IV-431-434.

[5] L.N. Alves, R.L. Aguiar, D.M. Santos "A CMOS Signal to Noise Measurement Circuit for Wireless Applications", Jornadas de Engenharia em Telecomunicações e Computadores, ISEL, Lisbon, Oct. 99.

[6] A.M. Tavares, R.T. Valadas, A. de Oliveira Duarte, "Design and Implementation of a Maximal ratio angle-diversity Receiver for Optical Wireless Communication Systems.”, Wireless Techn. and Systems: Millimeter-wave and Optical, Proc. of SPIE, vol.3232, Dallas, Texas, Nov. 5, 97.

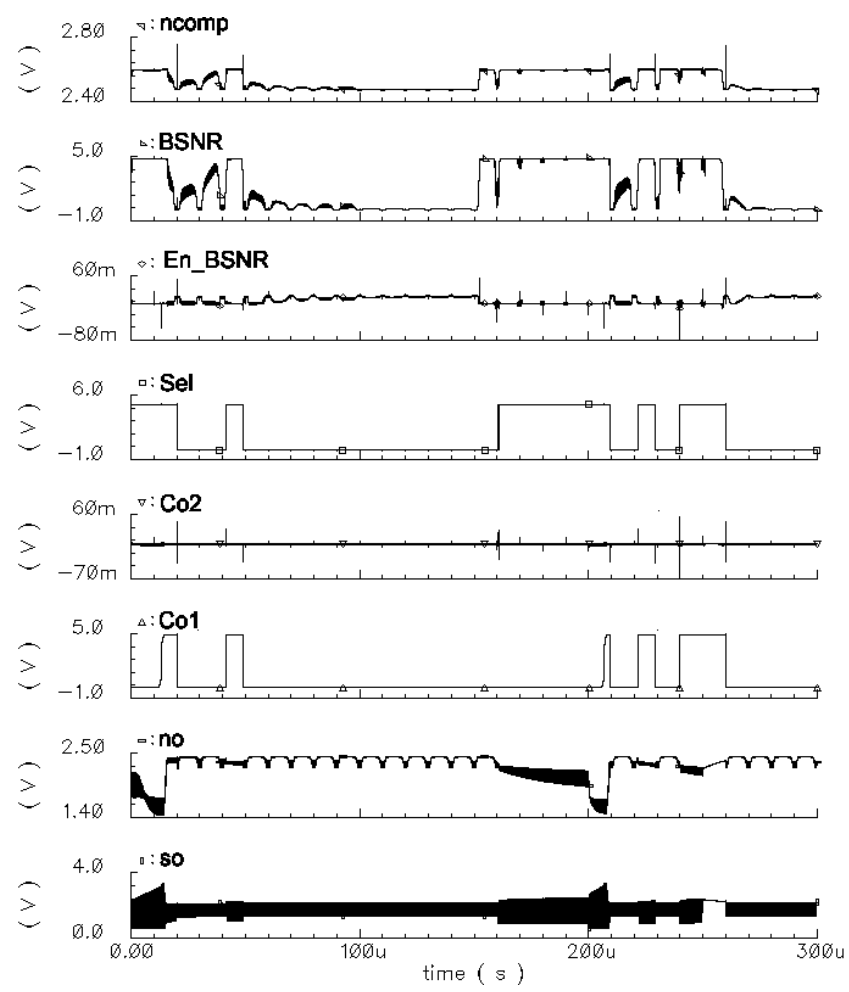

Fig. 10 - Sectorization: outputs 